\title{
Study of Genetic Variability and Correlations in a Mutant Population of Groundnut
}

\author{
Venkatesh $^{1 *}$, A.G. Vijaykumar ${ }^{1}$, B.N. Motagi ${ }^{1}$ and R.S. Bhat ${ }^{2}$ \\ ${ }^{1}$ Department of Genetics and Plant Breeding, University of Agricultural Sciences, \\ Dharwad, India \\ ${ }^{2}$ Department of Biotechnology, University of Agricultural Sciences, Dharwad-580 005, \\ Karnataka, India \\ *Corresponding author
}

Keywords

Variability, Correlation, Yield, Heritability

Article Info

Accepted:

12 December 2018

Available Online:

10 January 2019

\section{A B S T R A C T}

A mutant population comprising of 42 primary mutants, 7 secondary mutants and 4 tertiary mutants along with the parent Dharwad Early Runner (DER) and eight most popular groundnut varieties were evaluated during kharif 2012 for various agronomic, traits and for resistance to rust and late leaf spot. The genotypes showed significant genotypic differences for all the quantitative and nutritional traits studied. They also differed significantly for rust and LLS resistance except for LLS at 70 DAS. Phenotypic coefficient of variation (PCV) and genotypic coefficient of variation (GCV) revealed high variability for number of pods/plant and pod yield/plant $(\mathrm{g})$. LLS and rust resistance at three stages exhibited moderate variability. Number of pods/plant $(\mathrm{g})$ and pod yield/plant $(\mathrm{g})$ also showed very high heritability and genetic advance over mean. Moderately high heritability was observed for LLS and rust resistance at 80 and 90 DAS when compared to 70 DAS. Pod yield/plant (g) showed positive and significant phenotypic and genotypic correlation with number of pods/plant, shelling percentage, test weight (g), SMK (\%) and pod length $(\mathrm{cm})$. Pod yield/plant $(\mathrm{g})$ showed negative but significant correlation both at phenotypic and genotypic level with scores taken at all the three stages of LLS and rust disease development. The association analyses between stages (70, 80 and 90 DAS) showed positive and significant phenotypic correlation for LLS and rust resistance. However, the association between LLS and rust resistance across the stages was not significant. Pod yield per plant (g) can be considered as a tool in selection programme to enhance groundnut productivity, as it showed high heritability coupled with high genetic advance over mean (GAM) and positive association with productivity traits.

\section{Introduction}

The cultivated groundnut (Arachis hypogaea L.) is one of the major and important oilseed crop of the world. Among various oilseeds, groundnut is unique in that it can be consumed directly as an item of food and also utilized in diverse ways viz., source of oil and preparation of value added food products. Further its protein-rich meal and fodder for livestock are added advantages to the farming community. With about 26 per cent protein, 48 per cent oil and 3 per cent fibre and higher calcium, thiamine and niacine contents, it has the potential to be used as a highly economical food supplement to fight malnutrition that 
occurs due to deficiencies of these nutrients in the cereal grains.

Efficiency of the selection is dependent upon the nature, extent and magnitude of the genetic variability present in the material and the extent to which it is heritable. Correlations provide estimates of magnitude and direction of association between the traits. Hence, an attempt was undertaken to assess the variability and association between the important traits in diverse mutant.

\section{Materials and Methods}

The study employed a mutant population consisting 42 primary mutants, 7 secondary mutants, 4 tertiary mutants, parents and eight popular varieties representing various subspecies and botanical varieties.

All the primary mutants originated upon mutagenesis with ethyl methane sulphonate (EMS) $(0.5 \%)$ from Dharwad Early Runner (DER). DER was recovered from a cross involving two fastigiata cultivars, viz. Dh 320 and CGC-1 (Gowda et al., 1989). Secondary mutants were obtained from a few primary mutants upon mutagenesis. However, spontaneous mutations in the secondary mutants gave rise to tertiary mutants.

The experiment was carried out in randomized complete block design with two replications during kharif season (2012) at the IABT Garden, Main Agricultural Research station, Dharwad. The replicated data of all the traits were subjected for statistical analysis viz., Analysis of variation (ANOVA), mean, range, genetic variability components such as phenotypic coefficient of variation (PCV), genotypic coefficient of variation (GCV), heritability and genetic advance as per cent mean (GAM) and correlation analysis. Statistical package Windostat Version 8.1was used for the analysis.

\section{Results and Discussion}

The genotypes were evaluated in field during kharif 2012 for agronomic and productivity traits along with their reaction to LLS and rust. The genotypes showed significant differences for all the agronomic and productivity traits (Table 1a) except resistance to LLS at 70 DAS (Table 1b).

The improvement of character in a population is a function of variability existing in the population. Hence, formulation of objectives in breeding programme should be essentially accompanied with the assessment of existing variability in the segregating populations. Phenotypic coefficient of variation (PCV) and genotypic coefficient of variation (GCV) revealed high variability for number of pods per plant and pod yield per plant(g), and moderate variability for other traits Rao (2016) and Bhargavi et al., (2017) (Table 2a). LLS and rust resistance at 70, 80 and 90 DAS, exhibited moderate variability (Table 2b). While high variability was recorded with the results published by Khedikar et al., (2008), Reddy and Gupta (1992).

Number of pods per plant and pod yield per plant (g) also showed very high heritability and genetic advance over mean, indicating the scope for selection among the genotypes. Similar reports were observed by Singh et al., (1996), Abhay-Darshora et al., (2002) and Shinde et al., (2010), Mukhesh et al., (2014) and Balaraju and Kenchangoudar (2016). $\operatorname{SMK}(\%)$, test weight(g) and pod length showed high heritability though they had moderate level of variability Rao (2016), Bhargavi et al., (2017) and Yusuf et al., (2017) (Table 2a). Moderately high heritability was observed for LLS and rust resistance at 80 and 90 DAS compared to 70 DAS (Table 2b). Correlation coefficients were computed to assess the magnitude and direction of association between the traits. 
Table.1a ANOVA for agronomic traits among mutant population and check varieties of groundnut

\begin{tabular}{|c|c|c|c|c|c|c|c|c|c|c|c|c|c|}
\hline Source of variation $\mathrm{df}$ & $\begin{array}{l}\text { Plant } \\
\text { height } \\
\text { (cm) }\end{array}$ & $\begin{array}{c}\text { Primary } \\
\text { branch } \\
\text { length }(\mathrm{cm})\end{array}$ & \begin{tabular}{|l|} 
No. of \\
primary \\
branches
\end{tabular} & $\begin{array}{c}\text { No. of } \\
\text { secondary } \\
\text { branches }\end{array}$ & $\begin{array}{c}\text { Leaf } \\
\text { Length } \\
(\mathrm{cm})\end{array}$ & $\begin{array}{r}\begin{array}{c}\text { Leaf } \\
\text { width } \\
(\mathrm{cm})\end{array} \\
\end{array}$ & $\begin{array}{c}\text { Shelling } \\
\text { percentage }\end{array}$ & $\begin{array}{c}\text { Sound } \\
\text { Mature } \\
\text { kernel }\end{array}$ & $\begin{array}{c}\text { Test } \\
\text { Weight } \\
\text { (g) }\end{array}$ & $\begin{array}{c}\text { Pod } \\
\text { t Length } \\
\text { (cm) }\end{array}$ & $\begin{array}{l}\text { Pod } \\
\text { Width } \\
\text { (cm) }\end{array}$ & $\begin{array}{c}\text { No. of } \\
\text { pods } \\
\text { per plant }\end{array}$ & $\begin{array}{c}\text { Pod } \\
\text { yield } \\
\text { per plant }(g)\end{array}$ \\
\hline Replications (rMSS) 1 & 0.12 & 24.80 & 4.65 & 0.07 & 1.07 & 0.00 & 16.33 & 1415.82 & 1.16 & 0.01 & 0.02 & 12.58 & 11.20 \\
\hline Genotypes (gMSS) 61 & $76.04 * *$ & $93.15 * *$ & $12.47 * *$ & $2.70 * *$ & $1.64 * *$ & $0.32 * *$ & $277.72 * *$ & $309.98 * *$ & $93.88 * *$ & $0.52 * *$ & $0.11 * *$ & $69.88 * *$ & $50.43 * *$ \\
\hline $\begin{array}{ll}\text { Error (eMSS) } & 61\end{array}$ & 4.74 & 13.76 & 1.22 & 0.02 & 0.30 & 0.14 & 78.82 & 70.32 & 31.39 & 0.04 & 0.05 & 4.03 & 2.26 \\
\hline $\mathrm{F}$ & 16.0 & 6.8 & 10.3 & 135.1 & 5.5 & 2.4 & 3.5 & 4.4 & 3.0 & 12.0 & 2.1 & 17.3 & 22.3 \\
\hline SEm \pm & 1.5 & 2.6 & 0.8 & 0.1 & 0.4 & 0.3 & 6.3 & 5.9 & 4.0 & 0.1 & 0.2 & 1.4 & 1.1 \\
\hline CV $(\%)$ & 7.7 & 10.9 & 15.5 & 9.8 & 10.0 & 13.9 & 17.3 & 10.3 & 17.6 & 7.4 & 17.5 & 13.3 & 15.1 \\
\hline $\mathrm{CD}(5 \%)$ & 4.4 & 7.4 & 2.2 & 0.3 & 1.1 & 0.7 & 17.8 & 16.8 & 11.2 & 0.4 & 0.5 & 4.0 & 3.0 \\
\hline
\end{tabular}

Table.1b ANOVA for reaction to LLS and rust among mutant population and check varieties of groundnut

\begin{tabular}{|c|c|c|c|c|c|c|c|}
\hline Source of variation & df & $\begin{array}{c}\text { Late leaf spot } \\
\text { at } 70 \text { DAS }\end{array}$ & $\begin{array}{c}\text { Late leaf spot } \\
\text { at } 80 \text { DAS }\end{array}$ & $\begin{array}{c}\text { Late leaf spot } \\
\text { at } 90 \text { DAS }\end{array}$ & $\begin{array}{c}\text { Rust } \\
\text { at } 70 \text { DAS }\end{array}$ & $\begin{array}{c}\text { Rust } \\
\text { at } 80 \text { DAS }\end{array}$ & $\begin{array}{c}\text { Rust } \\
\text { at } 90 \text { DAS }\end{array}$ \\
\hline Replications (rMSS) & 1 & 0.03 & 5.88 & 5.04 & 0.65 & 0.98 & 0.03 \\
\hline Genotypes (gMSS) & 61 & 0.47 & $1.63 * *$ & $2.97 * *$ & $0.46^{*}$ & $2.09 * *$ & $3.47 * *$ \\
\hline Error (eMSS) & 61 & 0.43 & 0.39 & 0.81 & 0.28 & 0.53 & 0.88 \\
\hline $\mathbf{F}$ & & 1.1 & 4.2 & 3.7 & 1.7 & 3.9 & 3.9 \\
\hline SEm \pm & & 0.5 & 0.4 & 0.6 & 0.4 & 0.5 & 0.7 \\
\hline CV (\%) & & 18.6 & 10.6 & 12.7 & 14.6 & 12.6 & 13.5 \\
\hline $\mathrm{CD}(5 \%)$ & & & 1.2 & 1.8 & 1.1 & 1.5 & 1.9 \\
\hline
\end{tabular}


Table.2a Estimates of genetic parameters for agronomic traits among mutant population and check varieties of groundnut

\begin{tabular}{|c|c|c|c|c|c|c|c|c|}
\hline \multirow[t]{2}{*}{ Traits } & \multirow[t]{2}{*}{ Mean } & \multicolumn{2}{|c|}{ Range } & \multirow{2}{*}{$\begin{array}{c}\text { PCV } \\
(\%)\end{array}$} & \multirow{2}{*}{$\begin{array}{c}\text { GCV } \\
(\%)\end{array}$} & \multirow{2}{*}{$\begin{array}{c}\mathbf{h}^{2} \text { (Broad } \\
\text { Sense) } \\
(\%)\end{array}$} & \multirow[t]{2}{*}{ GA } & \multirow[t]{2}{*}{ GAM } \\
\hline & & Min & Max & & & & & \\
\hline Plant height (cm) & 28.39 & 15.06 & 42.30 & 18.04 & 13.11 & 53 & 0.25 & 19.61 \\
\hline Primary branch length (cm) & 33.95 & 21.56 & 64.57 & 13.86 & 4.46 & 10 & 0.10 & 2.96 \\
\hline No. of primary branches & 7.11 & 3.40 & 14.20 & 15.38 & 13.43 & 76 & 1.42 & 24.17 \\
\hline No. of secondary branches & 2.92 & 0.00 & 26.40 & 17.15 & 14.62 & 73 & 1.83 & 25.68 \\
\hline Leaf length $(\mathrm{cm})$ & 5.50 & 3.57 & 7.74 & 13.32 & 8.44 & 40 & 0.40 & 11.01 \\
\hline Leaf width $(\mathrm{cm})$ & 2.66 & 2.08 & 4.41 & 17.69 & 15.27 & 75 & 1.57 & 27.15 \\
\hline Shelling percentage & 51.36 & 22.00 & 72.00 & 18.95 & 16.36 & 75 & 2.02 & 29.09 \\
\hline Sound mature kernel & 81.77 & 19.00 & 96.50 & 9.31 & 9.15 & 97 & 1.96 & 18.52 \\
\hline Test weight (g) & 31.88 & 20.00 & 53.50 & 14.93 & 14.26 & 91 & 0.81 & 28.07 \\
\hline Pod length (cm) & 2.80 & 1.76 & 3.99 & 12.06 & 11.90 & 97 & 11.63 & 24.17 \\
\hline Pod width (cm) & 1.29 & 0.92 & 2.43 & 18.04 & 13.11 & 53 & 0.25 & 19.61 \\
\hline No. of pods per plant & 15.12 & 4.05 & 37.95 & 39.09 & 37.95 & 94 & 11.48 & 75.89 \\
\hline Pod yield per plant (g) & 9.98 & 1.40 & 30.63 & 50.33 & 49.18 & 96 & 9.88 & 99.02 \\
\hline
\end{tabular}

Table.2b Estimates of genetic parameters for LLS and rust resistance traits among mutant population and check varieties of groundnut

\begin{tabular}{|c|c|c|c|c|c|c|c|c|}
\hline \multirow[t]{2}{*}{ Traits } & \multirow[t]{2}{*}{ Mean } & \multicolumn{2}{|c|}{ Range } & \multirow{2}{*}{$\begin{array}{c}\text { PCV } \\
(\%)\end{array}$} & \multirow{2}{*}{$\begin{array}{c}\text { GCV } \\
(\%)\end{array}$} & \multirow{2}{*}{$\begin{array}{c}\mathbf{h}^{2} \\
\text { (Broad } \\
\text { sense) } \\
(\%)\end{array}$} & \multirow[t]{2}{*}{ GA } & \multirow[t]{2}{*}{ GAM } \\
\hline & & Min & $\operatorname{Max}$ & & & & & \\
\hline Late leaf spot at 70 DAS & 3.52 & 3.00 & 5.50 & 13.86 & 4.46 & 10 & 0.10 & 2.96 \\
\hline Late leaf spot at $80 \mathrm{DAS}$ & 5.88 & 4.00 & 8.00 & 15.38 & 13.43 & 76 & 1.42 & 24.17 \\
\hline Late leaf spot at 90 DAS & 7.10 & 4.00 & 9.00 & 17.15 & 14.62 & 73 & 1.83 & 25.68 \\
\hline Rust at 70 DAS & 3.60 & 3.00 & 5.00 & 13.32 & 8.44 & 40 & 0.40 & 11.01 \\
\hline Rust at 80 DAS & 5.78 & 4.00 & 7.50 & 17.69 & 15.27 & 75 & 1.57 & 27.15 \\
\hline Rust at 90 DAS & 6.95 & 4.00 & 9.00 & 18.95 & 16.36 & 75 & 2.02 & 29.09 \\
\hline
\end{tabular}


Table.3a Phenotypic and genotypic correlation coefficients for agronomic traits

\begin{tabular}{|c|c|c|c|c|c|c|c|c|c|c|c|c|c|}
\hline Traits & $\begin{array}{c}\text { Plant } \\
\text { height } \\
\text { (cm) }\end{array}$ & $\begin{array}{l}\text { Primary } \\
\text { branch } \\
\text { length } \\
(\mathrm{cm})\end{array}$ & $\begin{array}{c}\text { No. of } \\
\text { primary } \\
\text { branches }\end{array}$ & $\begin{array}{c}\text { No. of } \\
\text { secondary } \\
\text { branches }\end{array}$ & $\begin{array}{c}\text { Leaf } \\
\text { length } \\
\text { (cm) }\end{array}$ & $\begin{array}{c}\text { Leaf } \\
\text { width } \\
(\mathrm{cm})\end{array}$ & $\begin{array}{l}\text { Shelling } \\
\text { percentage }\end{array}$ & $\begin{array}{c}\text { Sound } \\
\text { mature } \\
\text { kernel }\end{array}$ & $\begin{array}{c}\text { Test } \\
\text { weight } \\
\text { (g) }\end{array}$ & $\begin{array}{c}\text { Pod } \\
\text { length } \\
\text { (cm) }\end{array}$ & $\begin{array}{c}\text { Pod } \\
\text { width } \\
(\mathrm{cm})\end{array}$ & $\begin{array}{l}\text { No. of } \\
\text { pods } \\
\text { per } \\
\text { plant }\end{array}$ & $\begin{array}{l}\text { Pod yield } \\
\text { per plant } \\
\text { (g) }\end{array}$ \\
\hline Plant height (cm) & 1.000 & $0.458 * *$ & $-0.224 *$ & $-0.322 * *$ & $0.363 * *$ & $0 * * .305$ & $0.245 * *$ & $0.200^{*}$ & $0.244 * *$ & $0.315 * *$ & 0.160 & 0.120 & $0.370 * *$ \\
\hline $\begin{array}{l}\text { Primary branch length } \\
\text { (cm) }\end{array}$ & $0.457 * *$ & 1.000 & -0.140 & 0.020 & $0.251^{* *}$ & $0.281 * *$ & -0.140 & 0.070 & $-0.233 * *$ & 0.110 & -0.170 & -0.010 & 0.030 \\
\hline No. of primary branches & $-0.242 *$ & -0.152 & 1.000 & $0.610 * *$ & $-0.455 * *$ & $-0.365 * *$ & $-0.275 * *$ & $-0.245^{* *}$ & $-0.100 * *$ & -0.040 & -0.150 & 0.100 & -0.200 \\
\hline $\begin{array}{l}\text { No. of secondary } \\
\text { branches }\end{array}$ & $-0.334 * *$ & 0.025 & $0.653 * *$ & 1.000 & $-0.500 * *$ & $-0.256 * *$ & $-0.497 * *$ & $-0.457 * *$ & $-0.311 * *$ & -0.090 & $-0.208^{*}$ & $-0.238 * *$ & $-0.410 * *$ \\
\hline Leaf length (cm) & $0.414 * *$ & $0.308 * *$ & $-0.519 * *$ & $-0.564 * *$ & 1.000 & $0.693 * *$ & 0.030 & 0.130 & -0.090 & 0.150 & $0.200 *$ & -0.100 & 0.170 \\
\hline Leaf width (cm) & $0.471 * *$ & $0.456 * *$ & $-0.454 * *$ & $-0.354 * *$ & $0.808 * *$ & 1.000 & -0.100 & -0.110 & -0.170 & 0.130 & 0.160 & $-0.303 * *$ & 0.020 \\
\hline Shelling percentage & $0.313 * *$ & $-0.245^{* *}$ & $-0.356^{* *}$ & $-0.580 * *$ & 0.128 & -0.155 & 1.000 & $0.579 * *$ & $0.439 * *$ & -0.040 & -0.010 & $0.483 * *$ & $0.570 * *$ \\
\hline Sound mature kernel & $0.245 * *$ & 0.072 & $-0.292 * *$ & $-0.516 * *$ & $0.228 *$ & -0.136 & $0.678 * *$ & 1.000 & $0.411 * *$ & -0.040 & -0.010 & $0.441 * *$ & $0.520 * *$ \\
\hline Test weight (g) & $0.311 * *$ & $-0.359 * *$ & -0.134 & $-0.374 * *$ & 0.009 & $-0.308 * *$ & $0.503 * *$ & $0.428 * *$ & 1.000 & $0.461 * *$ & $0.412 * *$ & 0.170 & $0.500 * *$ \\
\hline Pod length (cm) & $0.359 * *$ & 0.155 & -0.050 & -0.097 & 0.156 & 0.166 & -0.044 & -0.085 & $0.569 * *$ & 1.000 & $0.470 * *$ & -0.120 & $0.180^{*}$ \\
\hline Pod width $(\mathrm{cm})$ & $0.236 * *$ & $-0.229 *$ & $-0.223 * *$ & $-0.288 * *$ & $0.413 * *$ & $0.398 * *$ & -0.095 & -0.078 & $0.614 * *$ & $0.600 * *$ & 1.000 & -0.100 & 0.090 \\
\hline No. of pods per plant & 0.136 & 0.017 & 0.095 & $-0.245 * *$ & -0.110 & $-0.390 * *$ & $0.617 * *$ & $0.546 * *$ & $0.229 *$ & -0.129 & -0.147 & 1.000 & $0.600 * *$ \\
\hline Pod yield per plant (g) & $0.400 * *$ & 0.035 & $-0.235 * *$ & $-0.418 * *$ & $0.210^{*}$ & 0.057 & $0.648 * *$ & $0.594 * *$ & $0.568 * *$ & $0.194 *$ & 0.102 & $0.621 * *$ & 1.000 \\
\hline
\end{tabular}

Table.3b Phenotypic and genotypic correlation coefficients for LLS and rust diseases at 70, 80, 90 days after sowing (DAS)

\begin{tabular}{|l|c|c|c|c|c|c|}
\hline \multicolumn{1}{|c|}{ Traits } & $\begin{array}{c}\text { Late leaf } \\
\text { spot at 70 } \\
\text { DAS }\end{array}$ & $\begin{array}{c}\text { Late leaf } \\
\text { spot at 80 } \\
\text { DAS }\end{array}$ & $\begin{array}{c}\text { Late leaf } \\
\text { spot at 90 } \\
\text { DAS }\end{array}$ & $\begin{array}{c}\text { Rust } \\
\text { at 70 DAS }\end{array}$ & $\begin{array}{c}\text { Rust } \\
\text { at 80 DAS }\end{array}$ & $\begin{array}{c}\text { Rust 90 DAS } \\
\text { at 90 DA }\end{array}$ \\
\hline $\begin{array}{l}\text { Late leaf spot } \\
\text { at 70 DAS }\end{array}$ & 1.000 & $0.479 * *$ & $0.487 *$ & $0.273 * *$ & 0.060 & -0.010 \\
\hline $\begin{array}{l}\text { Late leaf spot } \\
\text { at 80 DAS }\end{array}$ & $0.865 * *$ & 1.000 & $0.789^{* *}$ & 0.140 & $0.273 * *$ & 0.130 \\
\hline $\begin{array}{l}\text { Late leaf spot } \\
\text { at 90 DAS }\end{array}$ & $0.796 * *$ & $0.939 * *$ & 1.000 & $0.198^{*}$ & 0.160 & 0.150 \\
\hline Rust at 70 DAS & $0.369 * *$ & $0.277 * *$ & $0.294 * *$ & 1.000 & $0.448 * *$ & $0.488 * *$ \\
\hline Rust at 80 DAS & -0.112 & $0.319 * *$ & $0.260 * *$ & $0.657 * *$ & 1.000 & $0.823 * *$ \\
\hline Rust at 90 DAS & -0.040 & 0.153 & $0.243 * *$ & $0.699 * *$ & $0.930 * *$ & 1.000 \\
\hline
\end{tabular}

Below diagonal genotypic correlation coefficients; Above diagonal phenotypic correlation coefficients; ** : Significance at $5 \%$ and $1 \%$ probability, respectively 
Table.3c Phenotypic and genotypic correlation coefficients for productivity, nutritional diseases resistance traits

\begin{tabular}{|c|c|c|c|c|c|c|c|c|c|c|c|}
\hline Traits & $\begin{array}{l}\text { Shelling } \\
\text { percentage }\end{array}$ & $\begin{array}{l}\text { Sound } \\
\text { mature } \\
\text { kernel }\end{array}$ & $\begin{array}{c}\text { Test } \\
\text { weight } \\
\text { (g) }\end{array}$ & $\begin{array}{c}\text { Late leaf } \\
\text { spot at } 70 \\
\text { DAS }\end{array}$ & $\begin{array}{c}\text { Late leaf } \\
\text { spot at } 80 \\
\text { DAS }\end{array}$ & $\begin{array}{c}\text { Late leaf } \\
\text { spot at } 90 \\
\text { DAS }\end{array}$ & $\begin{array}{l}\text { Rust } \\
\text { at } 70 \\
\text { DAS }\end{array}$ & $\begin{array}{l}\text { Rust } \\
\text { at } 80 \\
\text { DAS }\end{array}$ & $\begin{array}{l}\text { Rust } \\
\text { at } 90 \\
\text { DAS }\end{array}$ & $\begin{array}{l}\text { No. of } \\
\text { pods per } \\
\text { plant }\end{array}$ & $\begin{array}{c}\text { Pod yield } \\
\text { per plant } \\
\text { (g) }\end{array}$ \\
\hline Shelling percentage & 1.000 & $0.579 * *$ & $0.439 * *$ & -0.094 & -0.031 & -0.166 & 0.060 & 0.018 & -0.058 & $0.483 * *$ & $0.570 * *$ \\
\hline Sound mature kernel & $0.678 * *$ & 1.000 & $0.411 * *$ & -0.032 & $-0.181 *$ & -0.122 & 0.106 & -0.076 & -0.003 & $0.441 * *$ & $0.519 * *$ \\
\hline Test weight (g) & $0.503 * *$ & $0.428 * *$ & 1.000 & -0.174 & 0.043 & 0.010 & -0.080 & -0.013 & -0.105 & 0.174 & $0.500 * *$ \\
\hline Late leaf spot at 70 DAS & $-0.721 * *$ & -0.116 & -1.076 & 1.000 & $0.479 * *$ & $0.487 * *$ & $0.273^{*}$ & 0.057 & -0.012 & $-0.212 *$ & $-0.244 * *$ \\
\hline Late leaf spot at 80 DAS & -0.036 & -0.150 & 0.088 & $0.865^{* *}$ & 1.000 & $0.789 * *$ & 0.143 & $0.273 * *$ & 0.126 & $-0.234 * *$ & $-0.190 *$ \\
\hline Late leaf spot at 90 DAS & -0.176 & -0.116 & 0.053 & $0.796^{* *}$ & $0.939 * *$ & 1.000 & $0.198 *$ & 0.160 & 0.154 & $-0.258 * *$ & $-0.267 * *$ \\
\hline Rust at 70 DAS & -0.007 & $0.276^{* *}$ & -0.136 & $0.369 * *$ & $0.277 * *$ & $0.294 * *$ & 1.000 & $0.448 * *$ & $0.488 * *$ & -0.133 & $-0.299 * *$ \\
\hline Rust at 80 DAS & 0.099 & -0.101 & 0.014 & -0.112 & $0.319 * *$ & $0.260 * *$ & $0.657 * *$ & 1.000 & $0.823 * *$ & $0.290 * *$ & $-0.229 *$ \\
\hline Rust at 90 DAS & -0.032 & -0.012 & $-0.194 * *$ & -0.040 & 0.153 & $0.243 * *$ & $0.699 * *$ & $0.930 * *$ & 1.000 & $0.303 * *$ & $-0.312 * *$ \\
\hline No. of pods per plant & $0.617 * *$ & $0.546^{* *}$ & $0.229^{*}$ & $-0.611 * *$ & $-0.283 * *$ & $-0.323 * *$ & $-0.248 * *$ & $-0.373 * *$ & $-0.384 * *$ & 1.000 & $0.601 * *$ \\
\hline Pod yield per plant (g) & $0.648 * *$ & $0.594 * *$ & $0.568 * *$ & $-0.737 * *$ & $-0.225^{*}$ & $-0.313 * *$ & $-0.467 * *$ & $-0.269 * *$ & $-0.395 * *$ & $0.622 * *$ & 1.000 \\
\hline
\end{tabular}

Below diagonal genotypic correlation coefficients Above diagonal phenotypic correlation coefficients *, **: Significance at 5\% and $1 \%$ probability, respectively 
Pod yield per plant (g) showed positive and significant phenotypic and genotypic correlation with number of pods per plant, shelling percentage, test weight $(\mathrm{g})$, sound mature kernel per cent $(\%)$ and pod length (cm) (Table 3a). Similar results of significant positive association of number of pods with pod yield per plant was reported by Francis and Ramalingam (1997) Sarala and Gowda (1998) and Narasimhalu et al., 2012, Similar results of significant positive association of pod yield per plant with shelling percentage were reported by Abhay-Darshora et al., (2002) Mahalakshmi et al., (2005) and Wang et al., (2006). Similar results of significant positive association of pod yield per plant $(\mathrm{g})$ with test weight $(\mathrm{g})$ was reported by Channayya (2009) and Azharudheen (2010), While significant positive association of pod yield per plant with sound mature kernel per cent was reported by Francis and Ramalingam (1997) and Vasanthi et al., (2015). This indicates the importance of the number of pods per plant $(\mathrm{g})$, shelling percentage $(\%)$, test weight (g), sound mature kernel per cent and pod length $(\mathrm{cm})$ traits towards contribution to pod yield per plant (g). Selection for these traits will be more reliable to derive high yielding genotypes.

Pod yield per plant(g) showed negative but significant correlation both at phenotypic and genotypic level with disease scores at all the three stages of LLS and rust development as these foliar diseases reduce the photosynthetic activity of the plant (Table 3c). Similar results of significant negative association of pod yield per plant $(\mathrm{g})$ with disease score were reported by John et al., (2005) and Wang et al., (2006). The association analyses between stages (70, 80 and 90 DAS) showed positive and significant phenotypic correlation for LLS and rust resistance. However, the association between LLS and rust resistance across the stages was not significant (Table $3 b)$.
Results indicated that the trait pod yield per plant(g) showed higher heritability coupled with high genetic advance over mean and positive correlation with number of pods per plant, shelling percentage, test weight $(\mathrm{g})$, sound mature kernel $(\%)$ and pod length $(\mathrm{cm})$ it can be considered to be used in selection programmes to improve yield of groundnut.

\section{References}

Abhay Darshora, Nagada, A. K. and Dashora, A., 2002, Genetic variability and character association in Spanish bunch groundnuts. Res. on Crops, 3: 416-440.

Azharudheen, 2010, Evaluation of RILs for nutritional traits in groundnut (Arachis hypogaea L.) M.Sc. Thesis, Univ. Agric. Sci. Dharwad (India).

Balaraju, M. and Kenchanagoudar, P. V., 2016, Genetic variability for yield and its component traits in interspecific derivatives of groundnut (Arachis hypogaea L.). J. Farm Sci., 29(2): 172176.

Bhargavi, G., Satyanarayana R. V. and Narasimha, R. K. L., 2017, Genetic analysis for morphological, physiological, yield and yield attributes in groundnut (Arachis hypogaea L.). Indian J. Agric. Res., 51(4): 396-398.

Channayya, 2009, Induced genetic variability for yield and oil quality traits in groundnut (Arachis hypogaea L.). M.Sc. Thesis, Univ. Agril. Sci. Dharwad (India).

Francis, R. M. and Ramalingam, R. S., 1997, Character association and path alaysis in F2 population of groundnut. Journal of Oilseeds Research, 14(1): 11-14.

Khedikar Y. P., 2008, Molecular tagging and Mapping of resistance to late leaf spot and rust in Groundnut (Arachis hypogaea L.). Ph.D. Thesis, Uni. Agric. Sci., Dharwad (India).

John K., Vasnthi R. P., Venkateswarulu O. 
and Harinath Naidu P., 2005, Variability and correlation studies for quantitative traits in spanish bunch groundnut (Arachis hypogaea 1.) genotypes, Legume Res., 28(3): 189193.

Mahalakshmi, P., Manivannan, N. and Muralidharan, V., 2005, Variability and correlation studies in groundnut (Arachis hypogaea L.). Legume Res., 28(3): 194-197.

Mukhesh, K. M., Prashant, K. R., Arvind, K., Bazil, A. S. and Chaurasia, A. K., 2014, Study on genetic variability and seed quality of groundnut (Arachis hypogaea L.) genotypes. Int. J. Eme. Tech. Adv. Engi., 4(6): 818-823.

Narasimhulu, R., Kenchanagoudar, P.V. and Gowda, M. V. C., 2012, Study of genetic variability and correlations in selected groundnut genotypes. International J. Appl. Biol. Pharmaceut. Technol., 3 (1): 355-358.

Rao, V. T., 2016, Genetic variability, correlation and path coefficient analysis under drought in groundnut (Arachis hypogaea L.). Legume Res., 39(2): 319322.

Reddy, K. R. and Gupta, R. V. S., 1992, Variability and interrelationship of yield and its component characters in groundnut. J. Maharashtra Agric. Univ., 17: 224-226.
Sarala, B. S. and Gowda, M. V. C., 1998, Variability and correlation studies in segregating genotypes of intersubspecific crosses of groundnut (Arachis hypogaea L.). Crop Improvement, 25: 122-123.

Shinde, P. P., Khanpara, M. D., Vachhani, J. H., Jivani, L. L. and Kachhadia, V. H., 2010, Genetic variability in Virginia bunch groundnut (Arachis hypogaea L.). Plant Archives, 10(2): 703-706.

Singh, B. M., Das, S. S. and Srivastava, S., 1996, Variability for HPS grade groundnut in F4 generation. J. Appl. Biol., 6(1-2): 28-32.

Vasanthi, R. P., Suneetha, N. and Sudhakar, P. 2015, Genetic variability and correlation studies for morphological, yield and yield attributes in groundnut (Arachis hypogaea L.). J. Oilseeds Res., 38(1): 9-15.

Wang, C. T., Yang, X. D., Tang, Y. Y., Zhang, T. C., Xu, T. Z. and Lin, G. Z., 2006, EMS induced variations in pod characters of peanut. J. Peanut Sci., 2: 3-4.

Yusuf, Z., Zeleke, H., Mohammed, W., Hussein, S. and Hugo, A., 2017, Estimate of genetic variability parameters among groundnut (Arachis hypogaea L.) genotypes in Ethiopia. Int. J. Plant Breed. Crop Sci., 4(2): 225230.

\section{How to cite this article:}

Venkatesh, A. G. Vijaykumar, B. N. Motagi and Bhat, R.S. 2019. Study of Genetic Variability and Correlations in a Mutant Population of Groundnut. Int.J.Curr.Microbiol.App.Sci. 8(01): 1423-1430. doi: https://doi.org/10.20546/ijcmas.2019.801.151 